\title{
The Attitudes and Perceived Barriers Regarding the Practice of Evidence-Based Management Among Healthcare Managers in Saudi Arabia
}

Njoud Alsubaie

King Fahad Specialist Hospital in Dammam, Subaie2njoud@gmail.com

Bussma Bugis

Saudi Electronic University, bussma31311@yahoo.com

Follow this and additional works at: https://nsuworks.nova.edu/ijahsp

Part of the Health and Medical Administration Commons, and the Health Services Administration Commons

\section{Recommended Citation}

Alsubaie N, Bugis B. The Attitudes and Perceived Barriers Regarding the Practice of Evidence-Based Management Among Healthcare Managers in Saudi Arabia. The Internet Journal of Allied Health Sciences and Practice. 2021 Jan 01;19(2), Article 6.

This Manuscript is brought to you for free and open access by the College of Health Care Sciences at NSUWorks. It has been accepted for inclusion in Internet Journal of Allied Health Sciences and Practice by an authorized editor of NSUWorks. For more information, please contact nsuworks@nova.edu. 


\title{
The Attitudes and Perceived Barriers Regarding the Practice of Evidence-Based Management Among Healthcare Managers in Saudi Arabia
}

\begin{abstract}
Purpose: The study purpose was to explore attitudes and identify perceived barriers regarding the practice of evidence-based management (EBMgt) by healthcare managers. Method: A cross-sectional descriptive study was conducted using a simple random sample. The study population included middle- and lowerlevel healthcare managers at a specialized hospital in Saudi Arabia. A total of 165 healthcare managers were invited to complete a hard copy-survey. Results: The surveys were completed by 120 respondents, yielding a response rate of $72 \%$. The study results showed that most of the participants had a favourable attitude towards the practice of EBMgt. However, a lack of training programmes, a lack of time, limited understanding of scientific research, and a lack of organizational support and culture for implementing the EBMgt approach were perceived as major barriers to the practice of EBMgt by the respondents. Conclusions: The findings of this study provide policymakers and healthcare leaders with baseline information about barriers affecting the adoption of EBMgt among healthcare managers. Further research to explore effective strategies that might increase the use of evidence-based approaches and reduce barriers to the adoption of EBMgt is recommended.
\end{abstract}

\section{Author Bio(s)}

Njoud Alsubaie, MHA, is a Project Manager at King Fahad Specialist Hospital - First Health Cluster in Eastern Province of Saudi Arabia. She is also a certified medical coder.

Bussma Ahmed Bugis, DrPH, MPA, is an Assistant Professor of Health Services Organizations in the College of Health Sciences at the Saudi Electronic University. 


\title{
IIIAHSP \\ The Internet Joumnal of Allied Health Sciences and Practice \\ Dedicated to allied health professional practice and education
}

Vol. 19 No. 2 ISSN 1540-580X

\section{The Attitudes and Perceived Barriers Regarding the Practice of Evidence-Based Management (EBMgt) Among Healthcare Managers in Saudi Arabia}

\author{
Njoud Alsubaie ${ }^{1}$ \\ Bussma Ahmed Bugis ${ }^{2}$ \\ ${ }^{1}$ King Fahad Specialist Hospital \\ ${ }^{2}$ Saudi Electronic University
}

\begin{abstract}
Purpose: The study purpose was to explore attitudes and identify perceived barriers regarding the practice of evidence-based management (EBMgt) by healthcare managers. Method: A cross-sectional descriptive study was conducted using a simple random sample. The study population included middle- and lower-level healthcare managers at a specialized hospital in Saudi Arabia. A total of 165 healthcare managers were invited to complete a hard copy-survey. Results: The surveys were completed by 120 respondents, yielding a response rate of $72 \%$. The study results showed that most of the participants had a favourable attitude towards the practice of EBMgt. However, a lack of training programmes, a lack of time, limited understanding of scientific research, and a lack of organizational support and culture for implementing the EBMgt approach were perceived as major barriers to the practice of EBMgt by the respondents. Conclusions: The findings of this study provide policymakers and healthcare leaders with baseline information about barriers affecting the adoption of EBMgt among healthcare managers. Further research to explore effective strategies that might increase the use of evidence-based approaches and reduce barriers to the adoption of EBMgt is recommended.
\end{abstract}

KEYWORDS: Evidence-based management, healthcare manager, specialized hospital, Saudia Arabia 


\section{INTRODUCTION}

For the last 20 years, there has been an extensive effort to develop new managerial models and patterns within organizations, especially in healthcare organizations. ${ }^{1,2}$ Evidence is applied and used in medicine, nursing, economics, politics, management, and many other fields. In the healthcare management field, evidence is used and applied through a new managerial model, namely is evidence-based management (EBMgt). This model is used for managing healthcare organizations, especially hospitals. ${ }^{3}$ The changing healthcare field in recent years has complicated the role of hospital management. Increasing concerns about cost, quality, and access, rapid technology advances, higher expectations of consumers and intense competitive pressures are examples of complex challenges facing hospital management. ${ }^{4}$ As a result, the complexity of hospital management concerning skills and specialties has become a dominant issue. Therefore, managers are forced to consider EBMgt in their work to attain effective management and improve their competency in decision making. ${ }^{5}$

Healthcare management is "the profession that provides leadership and direction to organizations that deliver personal health services and to division, departments, units, or services within those organizations". ${ }^{6}$ In accordance, healthcare managers are professionals who lead and direct health organizations at different levels. Health organizations are different in size and complexity which require hiring healthcare managers at different levels. Managerial positions in healthcare organizations exist at lower, middle, and upper levels. Higher-level managerial positions have more authority and power than lower-level managerial positions. Upperlevel managers, also called senior managers, focus at the organizational level, while middle and lower healthcare managers focus on managing others of effective work. The position of the healthcare manager gives him/her the authority to shape the organization by conducting significant decisions. ${ }^{6}$ This study focused on middle and lower-level healthcare managers because of two reasons. First, the majority of healthcare managers are appointed at middle and lower levels. The second reason is that most of the internal tasks are done at these levels. While upper-level managers ensure wider organizational performance and viability. Many people tend to focus on the role of senior managers; however, it should be realized that healthcare management occurs at also other levels.

Healthcare managers are leaders with the authority to make decisions. Such decisions have a significant impact on the efficiency of the healthcare delivery process and the success of healthcare organizations. Ensuring the quality and safety of the delivered healthcare, cost efficiency and effectiveness requires the hospital manager to consider an evidence-based approach when making decisions. ${ }^{7}$ Evidence-based decision making would improve the competence of decision makers and enhance their motivation to implement more scientific techniques when making decisions. ${ }^{8}$ Moreover, it is considered one of the necessary competencies for the effective management of modern healthcare organizations and has proposed management competencies that include acquiring, appraising and implementing scientific research findings for management decisions. ${ }^{9}$ Healthcare managers should consider the efficiency of applying evidence-based decision-making strategies in their management process because of the considerable amendments of this approach. For instance, clinical guidelines and decision support system materials for healthcare professionals have become prolific due to the application of evidence-based medicines. ${ }^{10,11}$ However, EBMgt has been slowly adopted by healthcare managers, while other healthcare professionals have generally embraced the principles and practices of evidence-based medicine. ${ }^{12}$

The limited use of evidence-based practice could be a result of the perceived barriers by practitioners that prevent their ability to practice evidence-based approach. These barriers could be organizational barriers and limitations perceived in the work environment such as lack of time and limited support for implementing research or could be individual barriers such as limited understanding research methods and findings and lack of interest toward evidence-based practice..$^{13} \mathrm{It}$ is expected that managers are more likely to incorporate the best available evidence into their practice when they have a positive attitude towards scientific evidence and see the barriers to its use as surmountable. It is expected that managers are more likely to incorporate the best available evidence into their practice when they have a positive attitude towards scientific evidence and see the barriers to its use as surmountable. To enhance the uptake of EBMgt, it is important to explore health care managers' attitudes and perceived barriers related to the practice of EBMgt. ${ }^{14} \mathrm{~A}$ review of the literature indicates that very limited studies have been published regarding the current attitudes and perceived barriers of hospital managers regarding the practice of EBMgt in today's Saudi healthcare environment. Therefore, the goal of this study is to explore healthcare managers' attitudes towards practicing EBMgt and identify barriers that prevent the practice of EBMgt among healthcare managers at a specialized hospital in Saudi Arabia.

This study aims to achieve the following: identify source of evidence that healthcare managers consult in their daily practice, determine attitudes towards the practice of EBMgt among healthcare managers, and explore perceived barriers among healthcare managers that prevent the practice of EBMgt. 


\section{METHODs}

A cross-sectional, descriptive research method was utilized between October and November 2018. The targeted population was all 248 middle land lower-level healthcare managers within the hospital including 158 department heads (middle level managers) and 90 supervisors (lower-level managers). The participants were managers within the hospital from different departments: finance department, human resources, quality and strategic planning, information technology, laboratory, imaging, pharmacy, respiratory therapy, nursing, medical records, and many other departments. A simple random sampling was used in this study with a sample size of 187 healthcare managers (74 supervisors and 113 department heads). The sample size was calculated using Raosoft.com at a marginal error of $5 \%$ and a $95 \%$ confidence interval. Due to constraints and difficulties in reaching all participants, 165 surveys were distributed ( $88.2 \%$ of the sample size). Among the 165 surveys, 120 were collected, yielding a response rate of $72 \%$. The 120 participants were healthcare managers working at the selected hospital during the study period. However, 118 of them were fully affiliated with the hospital while two were not affiliated with the hospital as they might be affiliated with other entities and they were assigned for temporary assignments at the selected hospital.

The survey used for this study was a self-developed based on the study purposes and the literature review, then was validated through expert opinions. $3,7,15,16$ The survey included 28 closed-ended questions divided into four sections. First, Demographic characteristics were gathered through asking questions about age, gender, years of experience, administrative roles, position status (temporary or permanent), the highest level of education, and the familiarity of the EBMgt term. The second section measured the frequency of using sources of information when making decisions. In this section, each item was scored on a Likert scale ranging from 5 (always) to 1 (never). The third section was composed of questions about attitudes toward practicing EBMgt. Each item was presented in an affirmative statement and scored on a Likert scale ranging from 5 (strongly agree) to 1 (strongly disagree). The last section assessed the perceived barriers toward practicing EBMgt by healthcare managers. All the items presented in section 4 were negative statements. Questions listed in sections 3 and 4 were scored on a Likert scale ranging from 5 (strongly agree) to 1 (strongly disagree).

The paper-based surveys were distributed either by making an appointment with the departments' secretary or by direct contact with the participants through their emails or phone numbers. The surveys sent inside a closed envelop and contained an invitation letter to participation (cover letter) which included data about the purposes and the procedures of the study, indicating that participation in the study is voluntary, participants could withdraw freely for any purpose at any time during the survey and contact information of researchers. To ensure confidentiality and encourage honest and transparent responses, anonymity was insured. The surveys were sent and collected through enclosed envelops that contains investigators' contact information only. Respondents were requested not to indicate their personal details on the returned questionnaires.

\section{HUMAN SUBJECTS CONSIDERATIONS}

The study protocol has been reviewed and approved by the Institutional Review Board Office, National Registration Number $(\mathrm{H}$ 05-D002). The study was determined to be EXEMPT from review as study \# ITS0301.

\section{ANALYSIS AND RESULTS}

Following the completion of the survey, the data received were entered in Excel and then analyzed using SPSS version 25.0. The collected data from middle and lower-level managers where analyzed combined and there were no comparisons made between middle versus lower-level healthcare managers because the comparison between the two levels was not one of the study goals. Descriptive statistics were calculated for each item in the survey (response frequencies and percentages). The study results in Table 1 show the participants' characteristics. In addition, 48.35\% (58/120) were familiar with the concept of EBMgt. However, $41.7 \%$ (50/120) were unfamiliar with the concept, and 10\% (12/120) reported "maybe" with regard to familiarity with the concept.

Table 1: Characteristics of Participants ( $\mathrm{N}=120)$

\begin{tabular}{llcc}
\hline Characteristics & Classification & No. & Percentage \\
\hline Affiliation with the hospital & Yes & 118 & 98.3 \\
\cline { 2 - 4 } & No & 2 & 1.7 \\
\hline \multirow{2}{*}{ Gender } & Male & 60 & 50.0 \\
\cline { 2 - 4 } & Female & 60 & 50.0 \\
\hline \multirow{2}{*}{ Age } & $25-35$ & 37 & 30.8 \\
\cline { 2 - 4 } & $36-45$ & 64 & 53.4 \\
\hline
\end{tabular}




\begin{tabular}{llll}
\hline \multirow{2}{*}{$\begin{array}{l}\text { Years of experience in the } \\
\text { management field }\end{array}$} & $46-55$ & 19 & 15.8 \\
\cline { 2 - 4 } & $0-2$ & 12 & 10.0 \\
\cline { 2 - 4 } & $3-5$ & 31 & 25.8 \\
\cline { 2 - 4 } & $6-10$ & 37 & 30.8 \\
\cline { 2 - 4 } & $>10$ & 40 & 33.4 \\
\hline Status of the role & Head & 61 & 50.8 \\
\cline { 2 - 4 } & Supervisor & 59 & 49.2 \\
\hline Highest level of education & Permanent & 36 & 30.0 \\
\cline { 2 - 4 } & Temporary & 84 & 70.0 \\
\cline { 2 - 4 } & Doctoral degree & 9 & 7.5 \\
\cline { 2 - 4 } & Master's degree & 23 & 19.2 \\
\cline { 2 - 4 } & BSC & 72 & 60.0 \\
\cline { 2 - 4 } & Diploma & 16 & 13.3 \\
\hline Participants' background & Clinical & 41 & 34.2 \\
\cline { 2 - 4 } & Administrative & 34 & 37.5 \\
\cline { 2 - 4 } & Both & 12 & 28.3 \\
\hline Familiarity with EBMgt & Yes & 50 & 10.0 \\
\cline { 2 - 4 } & Maybe & & 41.7 \\
\cline { 2 - 4 } & No & &
\end{tabular}

Regarding to the sources of information used by healthcare managers when making decisions, Table 2 shows six types of sources listed in the survey. A five-point Likert scale was used to measure the frequency of using these sources when making decisions. The top four sources of information used by healthcare managers in this study are professional experience, organizational internal data, stakeholders' values and concerns and peer consultation.

Table 2: Sources of Information Used by Healthcare Managers (\%) ( $\mathrm{N}=120)$

\begin{tabular}{llccccc}
\hline & Sources & Never & Rarely & Sometimes & Often & Always \\
\hline 1 & Personal judgement & 6.7 & 19.1 & 33.3 & 21.7 & 19.2 \\
\hline 2 & Professional experience & 0 & 0 & 9.2 & 41.7 & 49.2 \\
\hline 3 & Peer consultation & 0 & 5.0 & 35.8 & 38.3 & 20.9 \\
\hline 4 & Scientific research & 5.8 & 12.5 & 30.0 & 32.5 & 19.2 \\
\hline 5 & Organizational data & 0.8 & 1.7 & 18.3 & 31.7 & 47.5 \\
\hline 6 & Stakeholders' values and expectations (employees and patients) & 1.7 & 6.6 & 21.7 & 30.8 & 39.2 \\
\hline
\end{tabular}

The results in Table 3 show that five items were listed in the survey to measure participants' attitudes towards the practice of EBMgt using a five-point Likert scale. Overall, the results showed that the majority of the participants had a positive attitude toward the practice of EBMgt. The frequency of participant responses was $0 \%$ for strongly disagree and very low for disagree (not more than .8\%). However, the frequency of neutral responses ranged between $4.2 \%$ and $17.5 \%$. Table 3 also shows that the majority of the respondents have a favourable attitude towards the practice of EBMgt. 
Table 3: Attitudes Towards the Practice of EBMgt (\%) ( $\mathrm{N}=120)$

\begin{tabular}{|c|c|c|c|c|c|c|}
\hline & Attitudes & $\begin{array}{l}\text { Strongly } \\
\text { Disagree }\end{array}$ & Disagree & Neutral & Agree & $\begin{array}{l}\text { Strongly } \\
\text { Agree }\end{array}$ \\
\hline 1 & $\begin{array}{l}\text { It is important for healthcare managers } \\
\text { to make decisions based on the best } \\
\text { evidence }\end{array}$ & 0 & 0.8 & 5.8 & 50.0 & 43.4 \\
\hline 2 & $\begin{array}{l}\text { I support the adoption of EBMgt in } \\
\text { healthcare management }\end{array}$ & 0 & 0 & 10.8 & 56.7 & 32.5 \\
\hline 3 & $\begin{array}{l}\text { Employing EBMgt improves an } \\
\text { organization's performance }\end{array}$ & 0 & 0 & 17.5 & 50.0 & 32.5 \\
\hline 4 & $\begin{array}{l}\text { EBMgt increases the quality of my } \\
\text { management decisions. }\end{array}$ & 0 & 0.8 & 10.8 & 51.7 & 36.7 \\
\hline 5 & $\begin{array}{l}\text { I am interested in learning or } \\
\text { improving EBMgt skills }\end{array}$ & 0 & 0 & 4.2 & 50.0 & 45.8 \\
\hline
\end{tabular}

Finally, Table 4 shows that seven barriers to practising EBMgt were listed in the survey on a five-point Likert scale. The results indicated that the top four barriers to the use of EBMgt were a lack of training, a lack of time, limited understanding of scientific research, and a lack of organizational support and culture for implementing the EBMgt approach.

Table 4: Perceived Barriers to the Practice of EBMgt (\%) $(\mathrm{N}=120)$

\begin{tabular}{|c|c|c|c|c|c|c|}
\hline & Barriers & $\begin{array}{l}\text { Strongly } \\
\text { Disagree }\end{array}$ & Disagree & Neutral & Agree & $\begin{array}{l}\text { Strongly } \\
\text { Agree }\end{array}$ \\
\hline 1 & Lack of time & 2.6 & 10.8 & 18.3 & 52.5 & 15.8 \\
\hline 2 & Lack of interest in EBMgt & 3.4 & 37.5 & 27.5 & 25.8 & 5.8 \\
\hline 3 & $\begin{array}{l}\text { Lack of organizational support } \\
\text { and culture }\end{array}$ & 0.8 & 15.0 & 19.2 & 52.5 & 12.5 \\
\hline 4 & Lack of resources & 3.3 & 26.7 & 16.7 & 40.8 & 12.5 \\
\hline 5 & $\begin{array}{l}\text { Lack of EBMgt training } \\
\text { programmes. }\end{array}$ & 0 & 6.6 & 9.2 & 46.7 & 37.5 \\
\hline 6 & $\begin{array}{l}\text { Limited understanding of } \\
\text { scientific research }\end{array}$ & 0.8 & 14.2 & 18.3 & 49.2 & 17.5 \\
\hline 7 & $\begin{array}{l}\text { Application of EBMgt is not } \\
\text { rewarded in the hospital }\end{array}$ & 0.8 & 13.3 & 27.6 & 35.8 & 22.5 \\
\hline
\end{tabular}

\section{DISCUSSION}

In this study, we explored sources of information used for decision making, and we identified attitudes and perceived barriers toward the practice of EBMgt among middle-and lower-level management. The majority of the participants were working at the selected hospital (98.3\%). Of the participants, $50.8 \%$ were department heads, and $49.2 \%$ were supervisors. Regarding to demographic characteristics such as age, the majority of the participants were $36-45$ years old (53.4\%). Regarding education level, the common degree held by healthcare managers was a bachelor's degree. Furthermore, the majority of the participants had an administrative background. The familiarity with the concept of EBMgt seems to be moderate among participants, as only $48.3 \%$ reported that they were familiar with the concept.

Regarding the information sources for decision making used by healthcare managers, according to the principles of EBMgt, the best sources of evidence are professional experience, scientific research, peer consultation, internal organizational data and stakeholders' values and concerns. ${ }^{17-20} \mathrm{~A}$ five-point Likert scale was used to measure the frequency of using information sources among participants. The findings suggest that personal experience (90.9\%), organizational internal data $(79.2 \%)$, stakeholders' values and concerns $(70 \%)$ and peer consultation (59.2\%) are the top four sources of information used by healthcare managers, 
as the majority of participants reported that they often used them. However, in Janati's study, most healthcare managers based their decisions on scientific research (91\%), personal judgement $(68.75 \%)$ and peer consultation $(64 \%))^{3}$ Personal judgement is not considered to be a sound source of evidence for decision making. ${ }^{21}$ The results of this study showed that a minority of the participants (19.2\%) always used personal judgement in their decision making. This indicated that the healthcare managers who participated in this study are more aware of the best sources of information used for decision making. However, the findings of the present study indicated that approximately half of the participants (51.7\%) reported that they often base their decisions on scientific research, and $30 \%$ reported that they sometimes consult scientific research. In addition, $(18.3 \%)$ of the participants reported that they rarely or never consulted scientific research. This is consistent with the statement of Dopson and his colleagues that healthcare managers do not often consult scientific research. ${ }^{22}$

The available literature show that limited studies have been published to explore attitudes toward the use of EBMgt among healthcare managers in today's Saudi healthcare environment. There are some studies found in the U.S. literature that measure attitudes toward EBMgt. 7,8 The present study was conducted among 120 healthcare managers from a specialized hospital in Saudi Arabia. The results of this study indicated that most of the respondents showed a favourable attitude toward the practice of EBMgt. Five positive statements were listed using a five-point Likert scale (it is important to make decisions based on the best evidence; I support the adoption of EBMgt, employing EBMgt improves my organization's performance; EBMgt increases the quality of my management decisions; and I am interested in learning or improving the skills of EBMgt practice) which elicited agreement ranging between $82.5 \%$ and $95.8 \%$. This is supported by the results of a question listed in the survey that asked participants to describe their overall attitude toward EBMgt, as $99.2 \%$ reported a positive attitude. The present study findings were consistent with those obtained by Guo et al. but differed from those obtained by Kovner and Rundall. ${ }^{7,8}$ Guo et al. performed a survey among 48 hospital administrators to assess similar goals of the current study which showed similar results regarding the positive attitudes towards the EBMgt practice by healthcare administrators. ${ }^{7}$ However, the Saudi healthcare system, structure, and culture is different than the U.S. Healthcare. ${ }^{23}$ The originality of our study is the study sample and the location of Gue et al. study was done in Idaho State at the United Stated while our study was done in Saudi Arabia. ${ }^{7}$ On the other hand, Kovner and Rundall have conducted interviews among 68 healthcare managers. Kovner and Rundall's study revealed that healthcare managers had a negative attitude toward the practice of EBMgt which is the opposite of the current study results. ${ }^{8}$ This study indicated that the practice of EBMgt has been accepted by the participating healthcare managers. Also, healthcare management is a challenging and fast-paced field influenced by the movement of evidence-based practice (EBP). Increasingly more healthcare managers realize the importance of considering evidence when making decisions.

However, the findings from this study indicated that a majority of the participants have a positive attitude towards the practice of EBMgt. Nonetheless, perceived barriers that prevent the practice of EBMgt exist. The results of this study showed that a lack of training programmes, a lack of time, limited understanding of scientific research and a lack of organizational support and culture for implementing the EBMgt approach were the most perceived barriers. A lack of training programmes was found to be the major barrier indicated by respondents that prevented the implementation of the EBMgt approach, as $84.2 \%$ of participants either strongly agreed or agreed that a lack of training is a barrier. This is consistent with the findings of Guo et al. found that a lack of training was one of the top four barriers that prevented the practice of EBMgt among U.S. healthcare administrators. ${ }^{24}$ Researchers have suggested that training in EBMgt would increase healthcare managers' competencies in making better decisions using research evidence..$^{25,26}$ The second-leading barrier indicated by respondents in this study was the lack of time, which was reported by $68.3 \%$ of the participants. This finding is consistent with the outcomes of studies in management field, medicine and nursing, which have found that the limited time among practitioners was among the top barriers. ${ }^{15,27,28}$ Healthcare management is a complex profession, as it must consider the interests of the organization and patients. This keeps managers very busy, with no time to educate themselves on updated information in the management field. Pfeffer and Sutton stated it is difficult for managers to adopt EBMgt, as there is too much information for any manager to consume, and a lack of time is a major barrier to the use of EBMgt. ${ }^{19}$ Limited understanding of scientific research was perceived to be the third major barrier, as $66.7 \%$ of the participants reported it. This is consistent with the results of the study of Barend et al., as they indicated that the inability to interpret scientific research is an important barrier facing managers. ${ }^{15}$ In addition, a limited understanding of scientific research could be the reason why the participants in this study do not often consult this source. Janati et al. stated that to improve the utilization of EBMgt, hospital leaders need to promote a culture that allows managers to dedicate time to searching and consulting scientific evidence. ${ }^{3}$ However, a lack of organizational culture and support for implementing evidence-based decision making was found to be the fourth-leading barrier in this study, as $65 \%$ of participants reported this barrier. The same barrier was also identified in another study, where the lack of organizational culture and support was found to be a potential barrier experienced by healthcare managers. ${ }^{29} \mathrm{~A}$ perceived lack of rewards and incentives was reported to be a barrier by more than half of the participants. This is supported by previous studies in nursing and medicine. ${ }^{16,30}$ However, Humphries et al. stated that the use of evidence could be increased through the development and implementation of formal organizational processes for decision-making and organizational reward systems to encourage the use of evidence. ${ }^{29}$ 
Furthermore, a lack of organizational resources was shown to be a moderate barrier in this study, as $53.3 \%$ of participants reported this barrier. However, this finding differed from those of other studies that indicated that a lack of resources and limited access to scientific research were reported to be the major barriers. ${ }^{15,29}$ This could be because the hospital provides free access to online databases that contain a variety of articles from academic and scientific journals. Finally, a lack of interest in EBMgt was found to be a minor barrier perceived by participants in this study, as $31.6 \%$ either strongly agreed or agreed that they were not interested in EBMgt. This result is consistent with those found in the literature and supports the finding in this study that participants have positive attitudes towards the practice of EBMgt. ${ }^{16}$ Since EBMgt is an emerging approach, its practice among healthcare managers remains limited. Several factors exist at the organizational and personal level that prevent the adoption of EBMgt. To reduce these barriers, efficient ways of integrating evidence-based decision making into organizational processes are needed. The findings of this study provide policymakers and healthcare leaders with baseline information on barriers affecting the adoption of EBMgt among healthcare managers. In addition, the outcome of this study could be useful for researchers to explore possible interventions that might increase the use of evidence-based approaches and reduce perceived barriers to the adoption of EBMgt for better decision making among healthcare managers.

The aims of this study have been addressed. Sources of information for decision making were identified and attitudes and perceived barriers were explored. However, this study has some limitations. First, the generalizability of the research results may be limited due to the small sample size. Second, self- reporting may cause recall bias. There was no way to ensure the truth of the selfreported responses during the survey. Finally, as a range of managers of different departments were included, findings of this study may not be representative of specific professions.

Healthcare managers are accountable for their decisions. Decisions based on sound sources of information can have positive effects on the improvements in the performance of the healthcare organization. The findings of this study provide policymakers and healthcare leaders with baseline information about barriers affecting the adoption of EBMgt among healthcare managers. Strategies should be placed to reduce the barriers and enhance the uptake of EBMgt. Organizations' leaders need to create a climate that improves healthcare managers' awareness of scientific research and the use of scientific evidence as part of the decision-making process. Besides, effective training programs need to be developed to facilitate the practice of EBMgt and increase healthcare managers' knowledge and skills in appraising sound evidence and information. This will help in reducing barriers to the adoption of EBMgt. In medical and health sciences schools, EBP is one of the important in their curriculum. They teach the student how to search for evidence in online databases, how to evaluate evidence, and how to apply research evidence in their daily practice. ${ }^{23} \mathrm{It}$ is recommended that educational institutions that offer healthcare administration programs to include EBMgt in their curriculum to educate and prepare qualified healthcare leaders. Further studies are needed to manage the perceived barriers regarding the use of EBMgt among healthcare managers.

\section{CONCLUSIONS}

Healthcare managers must be held accountable for both hospitals and patients. Decisions based on sound sources of information can have positive effects on the performance of the healthcare organization. Understanding attitudes and perceived barriers regarding the use of EBMgt is a critical step in advancing EBMgt and increasing the uptake of research into healthcare management practice. We conducted this study on a sample of middle-and lower-level healthcare managers at a specialized hospital in Saudi Arabia. In addition, the study findings revealed that healthcare managers who were sampled often used sound sources of information for decision making. These sources include personal experience, and organizational data and stakeholders' values and concerns. However, the minority of those managers reported that they base their decisions on scientific research. Moreover, the study results indicated that most of the participants have a positive attitude towards the practice of EBMgt. However, a lack of training programmes, a lack of time and limited understanding of scientific research are perceived as major barriers to the practice of EBMgt. Notably, studies within other professions in which EBP is far more established report the same barriers. This suggests that healthcare managers tend to follow a pattern common among managers in other fields. 


\section{References}

1) Acton M. Evidence-based everything: some implications for community care. J Integr Care. 1998;6(5):189-192. doi:10.1108/14769018199800040

2) Baba V, HakemZadeh F. Toward a theory of evidence based decision making. Management Decision. 2012;50(5):832-867. doi:10.1108/00251741211227546

3) Janati A, Hasanpoor E, Hajebrahimi S, Sadeghi-Bazargani H. Health care managers' perspectives on the sources of evidence in evidence-based hospital management: A qualitative study in Iran. Ethiop J Health Sci. 2017;27(6):659. doi:10.4314/ejhs.v27i6.11

4) Ford-Eickhoff K, Plowman D, McDaniel R. Hospital boards and hospital strategic focus. Health Care Manage Rev. 2011;36(2):145-154. doi:10.1097/hmr.0b013e3182099f6e

5) Janati A, Hasanpoor E, Hajebrahimi S, Sadeghi-Bazargani H. Evidence-based management - healthcare manager viewpoints. Int J Health Care Qual Assur. 2018;31(5):436-448. doi:10.1108/ijhcqa-08-2017-0143

6) Buchbinder S, Shanks N. Introduction to Health Care Management. Burlington, MA: Jones \& Bartlett Learning; 2017.

7) Guo R, Hermanson PM, Farnsworth TJ. Study on hospital administrators' beliefs and attitudes toward the practice of evidence-based management. Hosp Top. 2016;94(3-4):62-66. doi:10.1080/00185868.2016.1258886

8) Kovner A, Rundall T. Evidence-based management reconsidered. Front Health Serv Manage. 2006;22(3):3-22. doi:10.1097/01974520-200601000-00002

9) Stefl M. Common competencies for all healthcare managers: the Healthcare Leadership Alliance Model. J Healthc Manag. 2008;53(6):360-373. doi:10.1097/00115514-200811000-00004

10) Sackett $D$, Rosenberg $W$, Gray J, Haynes R, Richardson W. Evidence based medicine: what it is and what it isn't. BMJ. 1996;312(7023):71-72. doi:10.1136/bmj.312.7023.71

11) Eddy D. Evidence-based medicine: a unified approach. Health Affairs. 2005;24(1):9-17. doi:10.1377/hlthaff.24.1.9

12) Heiwe $S$, Kajermo $K$, Tyni-Lenne $R$ et al. Evidence-based practice: attitudes, knowledge and behaviour among allied health care professionals. Int J Qual Health Care. 2011;23(2):198-209. doi:10.1093/intqhc/mzq083

13) Shifaza $F$, Evans $D$, Bradley $H$. Nurses' perceptions of barriers and facilitators to implement EBP in the Maldives. Advances in Nursing. 2014;2014:1-7. doi:10.1155/2014/698604

14) Frasure J. Analysis of instruments measuring nurses' attitudes towards research utilization: a systematic review. J Adv Nurs. 2007;61(1):5-18. doi:10.1111/j.1365-2648.2007.04525.x

15) Barends $E$, Villanueva J, Rousseau $D$ et al. Managerial attitudes and perceived barriers regarding evidence-based practice: An international survey. PLOS ONE. 2017;12(10):e0184594. doi:10.1371/journal.pone. 0184594

16) Bussières $A E$, Terhorst $L$, Leach $M$, Stuber $K$, Evans $R$, Schneider MJ. Self-reported attitudes, skills and use of evidencebased practice among Canadian doctors of chiropractic: a national survey. J Can Chiropr Assoc. 2015;59(4):332-348

17) Grundtvig M, Gullestad L, Hole T, Flønæs B, Westheim A. Characteristics, implementation of evidence-based management and outcome in patients with chronic heart failure: results from the Norwegian Heart Failure Registry. Eur J Cardiovasc Nurs. 2011;10(1):44-49. doi:10.1016/j.ejcnurse.2010.04.001

18) Francis-Smythe J, Robinson $L$, Ross $C$. The role of evidence in general managers' decision-making. Journal of General Management. 2013;38(4):3-21. doi:10.1177/030630701303800402

19) Willmer M. How nursing leadership and management interventions could facilitate the effective use of ICT by student nurses. J Nurs Manag. 2007;15(2):207-213. doi:10.1111/j.1365-2834.2007.00751.x

20) Slater H, Davies S, Parsons R, Quintner J, Schug S. A policy-into-practice intervention to increase the uptake of evidencebased management of low back pain in primary care: a prospective cohort study. PLOS ONE. 2012;7(5):e38037. doi:10.1371/journal.pone.0038037

21) Baack S. Book and Resource Reviews. Review of Pfeffer J \& Sutton RI Hard Facts, Dangerous Half-Truths \& Total Nonsense: Profiting From Evidence-Based Management. Academy of Management Learning \& Education. 2007;6(1):139149. doi:10.5465/amle.2007.24401712

22) Netscc.ac.uk. http://www.netscc.ac.uk/hsdr/files/project/SDO_FR_08-1808-242_V06.pdf. Published 2020. Accessed January 20, 2019.

23) Baranowski J. Health systems of the world--Saudi Arabia. Global Health--an Online Journal for the Digital Age. 2009;2(1) 
24) Guo R, Berkshire SD, Fulton LV, Hermanson PM. Factors affecting the adoption of evidence-based management among U.S. healthcare administrators. American Journal of Management. 2018;18(1):36-47. doi:10.33423/ajm.v18i1.303

25) Shortell S. Promoting evidence-based management. Front Health Serv Manage. 2006;22(3):23-30. doi:10.1097/01974520200601000-00003

26) Walshe K, Rundall T. Evidence-based management: from theory to practice in health care. Milbank Q. 2001;79(3):429-457. doi:10.1111/1468-0009.00214

27) van Dijk N, Hooft $L$, Wieringa-de Waard $M$. What are the barriers to residents' practicing evidence-based medicine? A systematic review. Acad Med. 2010;85(7):1163-1170. doi:10.1097/acm.0b013e3181d4152f

28) Solomons N, Spross J. Evidence-based practice barriers and facilitators from a continuous quality improvement perspective: an integrative review. J Nurs Manag. 2010;19(1):109-120. doi:10.1111/j.1365-2834.2010.01144.x

29) Humphries $S$, Stafinski T, Mumtaz $Z$, Menon $D$. Barriers and facilitators to evidence-use in program management: a systematic review of the literature. BMC Health Serv Res. 2014;14(1). doi:10.1186/1472-6963-14-171

30) Malik G, McKenna L, Plummer V. Facilitators and barriers to evidence-based practice: perceptions of nurse educators, clinical coaches and nurse specialists from a descriptive study. Contemp Nurse. 2016;52(5):544-554. doi:10.1080/10376178.2016.1188017 